\title{
A parsec-scale outflow from the luminous YSO IRAS 17527-2439
}

\author{
W. P. Varricatt
}

\author{
Joint Astronomy Centre, 660 N. Aohoku Pl., Hilo, HI-96720, USA \\ e-mail: w.varricatt@jach.hawaii.edu
}

Received 15 October 2010 / Accepted 17 December 2010

\begin{abstract}
Aims. We seek to understand the way massive stars form. The case of a luminous YSO IRAS 17527-2439 is studied in the infrared. Methods. Imaging observations of IRAS 17527-2439 are obtained in the near-IR JHK photometric bands and in a narrow-band filter centred at the wavelength of the $\mathrm{H}_{2}$ 1-0S(1) line. The continuum-subtracted $\mathrm{H}_{2}$ image is used to identify outflows. The data obtained in this study are used in conjunction with Spitzer, AKARI, and IRAS data. The YSO driving the outflow is identified in the Spitzer images. The spectral energy distribution (SED) of the YSO is studied using available radiative transfer models.

Results. A parsec-scale bipolar outflow is discovered in our $\mathrm{H}_{2}$ line image, which is supported by the detection in the archival Spitzer images. The $\mathrm{H}_{2}$ image exhibits signs of precession of the main jet and shows tentative evidence for a second outflow. These suggest the possibility of a companion to the outflow source. There is a strong component of continuum emission in the direction of the outflow, which supports the idea that the outflow cavity provides a path for radiation to escape, thereby reducing the radiation pressure on the accreted matter. The bulk of the emission observed close to the outflow in the WFCAM and Spitzer bands is rotated counter clockwise with respect to the outflow traced in $\mathrm{H}_{2}$, which may be due to precession. A model fit to the SED of the central source tells us that the YSO has a mass of $12.23 M_{\odot}$ and that it is in an early stage of evolution.
\end{abstract}

Key words. stars: formation - stars: pre-main sequence - ISM: jets and outflows - stars: protostars - circumstellar matter

\section{Introduction}

Low- and intermediate-mass stars are known to form by gravitational collapse and subsequent accretion of their parent molecular clouds, and driving collimated outflows. However, the main mechanism leading to the formation of massive stars is debated as to whether it is either disk accretion similar to that for lower mass stars (e.g. Yorke \& Sonnhalter 2002) or a merger of lowermass stars (e.g. Bonnell et al. 1998). Many of the recent CO line surveys show outflows from massive YSOs (e.g. Zhang et al. 2005; Beuther et al. 2002). Several massive YSO outflows have been observed in the near-IR, where the spatial resolution is better than that in single-dish $\mathrm{CO}$ line observations. A recent nearIR imaging survey by Varricatt et al. (2010) shows that massive stars up to at least late-O spectral types form primarily by disk accretion. The case of a luminous YSO taking birth by accretion is presented in this paper.

IRAS 17527-2439 (hereafter IRAS 17527) is a luminous YSO located in a dark cloud situated close to the Galactic plane $\left(l=4.8273^{\circ}, b=0.2297^{\circ}\right)$ in the Ophiuchus region. It is associated with emission from dense gas and dust typical of massive YSOs. Molinari et al. (1996) detected $\mathrm{NH}_{3}$ emission lines from this region. From the radial velocity of the $\mathrm{NH}_{3}$ lines $\left(V_{\mathrm{LSR}}=\right.$ $13.3 \mathrm{~km} \mathrm{~s}^{-1}$ ), they estimated a kinematic distance of $3.23 \mathrm{kpc}$, with the distance ambiguity resolved. Based on the IRAS colours they classified IRAS 17527 as a "high" source, which is possibly in a UCHII phase. (Note that at $12 \mu \mathrm{m}$, the IRAS catalogue gives only an upper limit flux for this source). In their 97.981-GHz CS(2-1) survey of UCHII candidates, Bronfman et al. (1996) detected IRAS 17527 at $V_{\mathrm{LSR}}=13.5 \mathrm{~km} \mathrm{~s}^{-1}$, similar to the velocity at which Molinari et al. (1996) detected $\mathrm{NH}_{3}$ emission.

Massive star formation is often associated with $\mathrm{H}_{2} \mathrm{O}$, $\mathrm{CH}_{3} \mathrm{OH}$ and $\mathrm{OH}$ maser emission. Palla et al. (1991) detected
$\mathrm{H}_{2} \mathrm{O}$ maser emission from IRAS 17527. From the radial velocity of the maser $\left(V_{\text {peak }}=-1.81 \mathrm{~km} \mathrm{~s}^{-1}\right)$ they estimated a kinematic distance of $17.9 \mathrm{kpc}$, which is quite different from the distance estimated from radial velocity of the dense gas tracers $\mathrm{NH}_{3}$ and CS. In massive YSOs, $\mathrm{H}_{2} \mathrm{O}$ maser is often considered to be excited in jets (e.g. Felli et al. 1992; Goddi et al. 2005). The blueshift of the wavelength of the maser emission in this region with respect to the velocity of the dense gas tracers indicates that $\mathrm{H}_{2} \mathrm{O}$ maser near IRAS 17527 also may be excited by a jet. Hence we adopt the distance estimate of $3.23 \mathrm{kpc}$ (Molinari et al. 1996) for the calculations in this paper. Surveys by van der Walt et al. (1995), Walsh et al. (1997) and Slysh et al. (1999) did not detect any $6.7 \mathrm{GHz}$ Class-II methanol maser from this region. Edris et al. (2007) detected faint (0.4 Jy) OH maser emission at $V_{\text {peak }}=11.53 \mathrm{~km} \mathrm{~s}^{-1}$, located $\sim 6^{\prime} \mathrm{NW}$ of IRAS 17527 . Nevertheless, this offset is less than their beam size. Therefore it remains to be investigated at better spatial resolution. The faintness of the detected $\mathrm{OH}$ maser is consistent with their observation that the $\mathrm{OH}$ masers associated with younger sources are weaker than the ones associated with HII or UCHII regions.

The VLA survey of Hughes \& MacLeod (1994) detected 6-cm emission from IRAS 17527 with a peak flux of $5.3 \mathrm{mJy}$. The source was however rejected as a UCHII candidate because the radio emission was diffuse.

\section{Observations and data reduction}

\subsection{UKIRT data}

Observations were carried out on 2010 Apr. 15 UT using the United Kingdom Infrared Telescope (UKIRT) and the Wide Field Camera (WFCAM; Casali et al. 2007). WFCAM has a pixel scale of $0.4^{\prime \prime}$ pix $^{-1}$ and employs four $2048 \times$ $2048 \mathrm{HgCdTe}$ HawaiiII RG arrays. Each array has a field of 


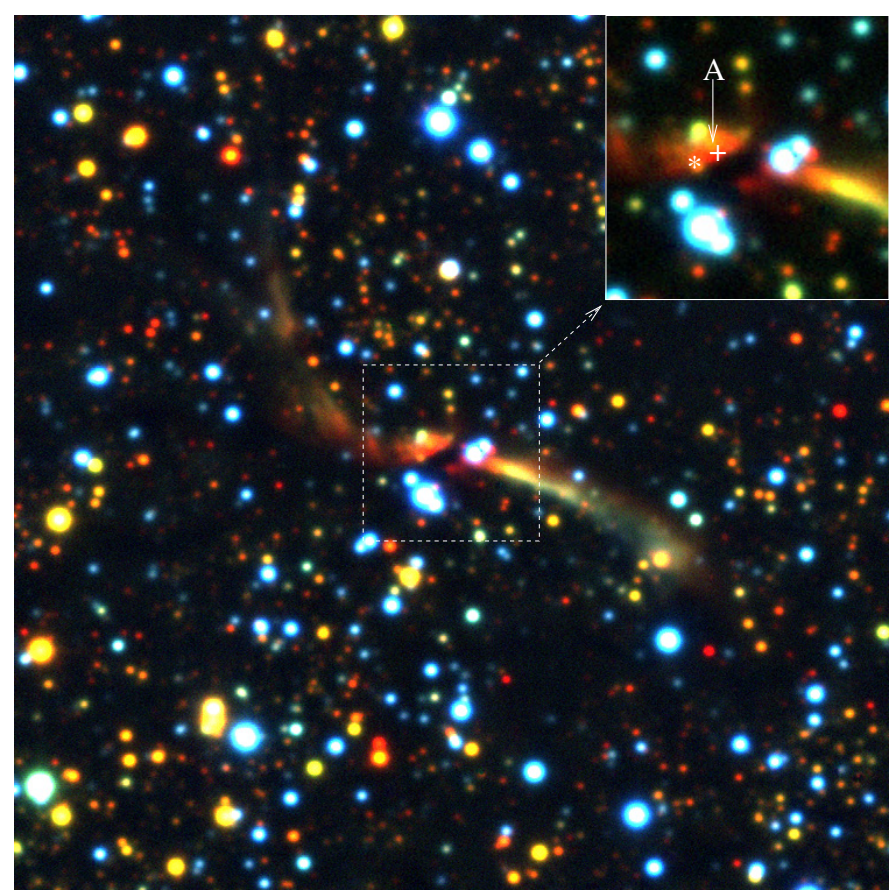

Fig. 1. WFCAM $J H K$ colour-composite image ( $J$-blue, $H$-green, $K$-red) in a $2.5^{\prime} \times 2.5^{\prime}$ field centred on IRAS 17527 . An expanded view of the central $0.5^{\prime} \times 0.5^{\prime}$ field is shown in the inset. "+" shows the location of the Spitzer source identified and "*” shows the IRAS position.

view of $13.65^{\prime} \times 13.65^{\prime}$. Observations were performed by dithering the object to 9 points separated by a few arcseconds and using a $2 \times 2$ microstepping. Hence the final pixel scale is $0.2^{\prime \prime}$ pixel $^{-1}$. Observations were obtained in $J, H$ and $K M K O$ filters and in a narrow-band $M K O$ filter centred at the wavelength of the $\mathrm{H}_{2}$ (1-0) $\mathrm{S} 1$ line at $2.1218 \mu \mathrm{m}$. The data were reduced by the Cambridge Astronomical Survey Unit (CASU); the archival and distribution of the data are carried out by the Wide Field Astronomy Unit (WFAU).

The sky conditions were not photometric during the observations. However, since the $K$ magnitude estimated here is from an average zero point calculated for a set of isolated point sources present in all dithered frames around IRAS 17527 and using their 2MASS magnitudes, the photometric quality is good. Figure 1 shows a $J H K$ colour composite image created from the WFCAM data in a $2.5^{\prime} \times 2.5^{\prime}$ field centred on IRAS 17527 .

The $\mathrm{H}_{2}$ image was continuum-subtracted using the $K$-band image. The average of the ratio of counts, for a few isolated point sources, between the $K$ and $\mathrm{H}_{2}$ images was obtained. The background-subtracted $K$-band image was scaled by this ratio and was then subtracted from the background-subtracted $\mathrm{H}_{2}$ image. In regions where the near-IR colours do not vary widely over the sources, this procedure subtracts out the extended emission well, and the point sources get subtracted out well when the seeing is similar for $K$ and $\mathrm{H}_{2}$ images. However, the $K / \mathrm{H}_{2}$ ratio has a dependence on the apparent near-IR colours. For objects like IRAS 17527, which are located in dark clouds in the galactic plane, the sources in the field are subjected to a wide range of interstellar extinction and several sources may have infrared excess due to circumstellar emission, resulting in a wide range of near-IR colours. Hence, for these regions, even when the seeing is stable, a perfect continuum-subtraction cannot be achieved for all objects in the field when we use a $K$-band image to subtract the continuum from the $\mathrm{H}_{2}$ image. Figure 2 shows the continuum-subtracted $\mathrm{H}_{2}$ image.

\subsection{Archival data}

This source was detected well by IRAS at 25 and $60 \mu \mathrm{m}$. At $12 \mu \mathrm{m}$, the IRAS flux density is only an upper limit. The flux density at $100 \mu \mathrm{m}$ is of poor quality and is affected by infrared cirrus. Hence the IRAS 12 and 100- $\mu$ m fluxes are not used in our analysis. IRAS 17527 was observed in the sky survey conducted by AKARI satellite (Murakami et al. 2007). In the mid-IR, the InfraRed Camera (IRC) on board AKARI detected a source at 9 and $18 \mu \mathrm{m}$. The AKARI Far-Infrared Surveyor (FIS) also detected a source in all 4 bands - at 65, 90, 140 and $160 \mu \mathrm{m}$.

The Spitzer space telescope observed IRAS 17527 as a part of the GLIMPSE II survey using the Infrared Array Camera (IRAC; Fazio et al. 2004) in bands 1-4, centred at 3.6, 4.5, 5.8 and $8.0 \mu \mathrm{m}$ respectively. Spitzer also observed this region using the Multiband Imaging Photometer for Spitzer (MIPS; Rieke et al. 2004) at $24 \mu \mathrm{m}$. The reduced Spitzer images and point source photometry in a $5^{\prime} \times 5^{\prime}$ field around IRAS 17527 were downloaded from the NASA/IPAC Infrared Science Archive. Figure 3 shows a colour composite image of a $5^{\prime} \times 5^{\prime}$ region constructed from the Spitzer images at 3.6, 4.5 and $8.0 \mu \mathrm{m}$.

\section{Results and discussion}

\subsection{The outflow}

Our near-IR images (Figs. 1, 2) reveal a spectacular outflow oriented NE-SW associated with IRAS 17527. A source with large near-IR colours is labelled "A" (see Sect. 3.2) in Fig. 1. The continuum-subtracted $\mathrm{H}_{2}$ image (Fig. 2) shows the outflow in $\mathrm{H}_{2}$ line emission; a dashed line (labelled "1") is drawn in Fig. 2 to guide the eyes. The outflow " 1 " has a length of 48 " in the SW and $34^{\prime \prime}$ in the NE if the outflow source is located at or near "A". This length corresponds to $0.75 \mathrm{pc}$ in the SW, at a distance of $3.23 \mathrm{kpc}$. The outflow is seen in $\mathrm{H}_{2}$ to be bent in an "S"shaped fashion, suggesting a precession of the jet. The direction of the outflow measured from the $\mathrm{H}_{2}$ images is $\sim 68^{\circ}$ East of North at the base. The detection of a well defined outflow suggests that the YSO associated with IRAS 17527 is taking birth through disk accretion. There is also tentative detection of two additional $\mathrm{H}_{2}$ emission features close to IRAS 17527, which are enclosed in ellipses in Fig. 2 and labelled " $2 a$ " and " $2 b$ "; this needs to be verified by deeper $\mathrm{H}_{2}$ imaging. If a second bipolar outflow is producing " $2 \mathrm{a}$ " and " $2 \mathrm{~b}$ ", it may be at an angle of $129^{\circ}$. Thus, the possibility of a companion needs to be explored through deeper $\mathrm{H}_{2}$ imaging and through imaging in the near- and mid-IR at higher angular resolution. The model fit to the SED (see Sect. 3.2.1) gives an angle of inclination of the disk axis with respect to the line of sight to be $18^{\circ}$, which implies a similar line-of-sight inclination for the outflow. The comparable brightness for the two outflow lobes of " 1 " seen in $\mathrm{H}_{2}$ suggests a higher inclination (even though it could be argued that this is mainly due to non-uniform distribution of exciting material rather than higher inclination). The outflows " 1 " and " $2 \mathrm{a}+2 \mathrm{~b}$ " are assigned names MHO 2147 and MHO 2148 respectively in the catalog of Molecular Hydrogen emission-line Objects $^{1}$ (Davis et al. 2010). Some additional line emission features detected in the continuum-subtracted $\mathrm{H}_{2}$ image are circled

${ }^{1}$ http://www.jach.hawaii.edu/UKIRT/MHCat/ 


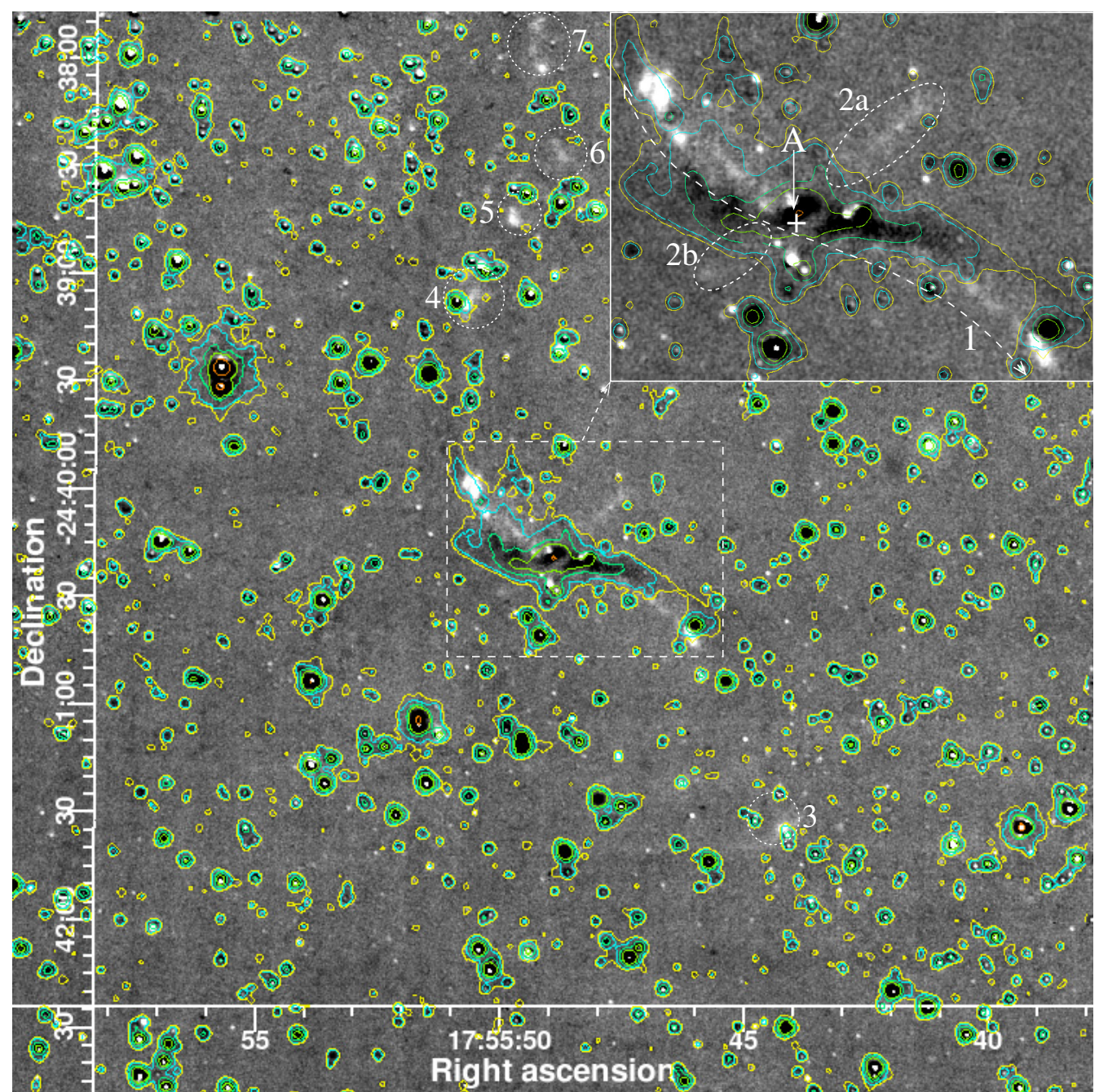

Fig. 2. The continuum-subtracted 2.122- $\mu \mathrm{m}$ image of IRAS 17527 over a $5^{\prime} \times 5^{\prime}$ field, smoothed with a 2-pixel $F W H M$ Gaussian to enhance the appearance of the faint emission features. "+" shows the location of the outflow source derived from the Spitzer 5.8- $\mu \mathrm{m}$ image. The contours generated from the Spitzer 4.5- $\mu$ m image are overlaid. An enlarged view of the central region is shown in the inset. The dashed line "1" shows the direction of the main outflow detected here. The ellipses " $2 a$ " and " $2 \mathrm{~b}$ " enclose $\mathrm{H}_{2}$ emission features which are probably due to a second outflow. Additional $\mathrm{H}_{2}$ emission features detected are circled and labelled " $3-7$ ". The image scale is $0.2^{\text {" }}$ pix $^{-1}$.

and labelled " $3-7$ " in Fig. 2. It is not clear if these are produced by outflow " 1 " or by different outflows.

There is a significant amount of emission along the outflow in our $J H K$ and unsubtracted $\mathrm{H}_{2}$ images and in the Spitzer-IRAC images. A major portion of the emission seen along the outflow in the $\mathrm{H}_{2}$ image is subtracted out upon continuum-subtraction and Fig. 2 shows only a chain of line emission knots. This suggests the presence of a large amount of continuum emission in the direction the outflow. Continuum emission close to the outflow is likely to be due to radiation from the YSO escaping through the outflow cavity. It has been proposed before that in massive star formation, the optically thin cavity carved out by the bipolar outflow may provide a path for radiation from the central source to escape, which reduces the radiation pressure on the accreted matter and aids the growth of a massive star. (Krumholz et al. 2005).

Figure 2 exhibits negative residuals along the bright regions of emission in the near-IR images, seen as dark lanes close to the outflow traced by the $\mathrm{H}_{2}$ knots labelled "1". The negative residuals close to the outflow are likely to be caused by large near-IR colours arising from extinction or excess or both, as discussed in Sect. 2.1. The contours generated from the Spitzer-IRAC 4.5- $\mu \mathrm{m}$ image are over-plotted in Fig. 2. Away from the outflow centre, the contours of the faint regions of the $4.5-\mu \mathrm{m}$ emission trace the outflow detected in $\mathrm{H}_{2}$ in the NE, but are rotated counterclockwise with respect to the $\mathrm{H}_{2}$ knots in the SW. Close to the base of the outflow, the contours of the bright regions of the $4.5-\mu \mathrm{m}$ emission are nearly EW. The $4.5-\mu$ m contours trace the bright regions of the $K$-band emission reasonably well; close to the base of the outflow, the contours of the bright regions of the $4.5-\mu \mathrm{m}$ emission have a slight counterclockwise rotation with respect to the bright regions of the emission in $K$ in the east. In general, the bright regions of emission seen in the near-IR and Spitzer images, close to the outflow, are rotated counter-clockwise with respect to the direction of the outflow implied by the presumably shock-excited $\mathrm{H}_{2}$ emission knots in "1". This rotation, again, is likely to be due to the precession of the jet, but needs to be thoroughly investigated. The brightest region of this emission in the near IR towards the SW lobe of the outflow is seen throughout the 1-8 $\mu \mathrm{m}$ wavelength range. It should be noted that transitions of $\mathrm{H}_{2}$ are present throughout the 1-8 $\mu \mathrm{m}$ wavelength range discussed here (Smith 1995; Neufeld et al. 2009). Spectroscopy in the near- and mid-IR will enable us to understand if the emission seen in in these bands close to the outflow "1" are line- or 


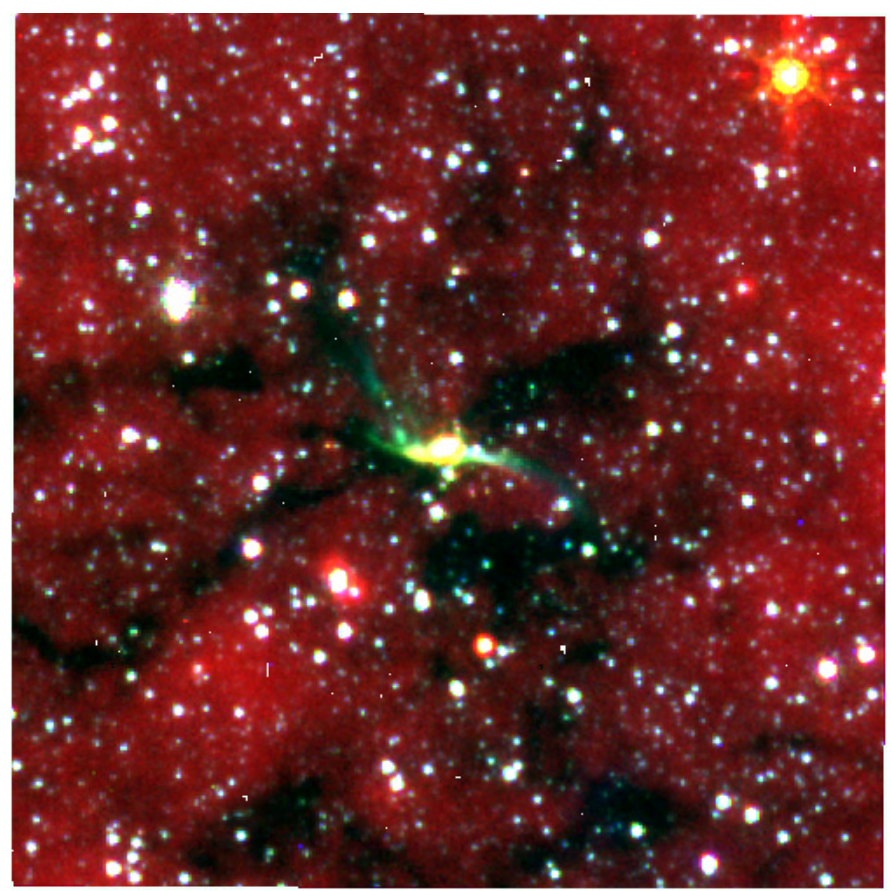

Fig. 3. A colour-composite image produced from Spitzer-IRAC data adopting blue, green and red, respectively for the 3.6, 4.5 and $8.0-\mu \mathrm{m}$ images. The image covers a $5^{\prime} \times 5^{\prime}$ field centred on IRAS 17527 .

continuum-dominated. If continuum-dominated, the emission, especially in short wavelengths like $J$, will imply radiation escaping from the hot central source.

\subsection{The source driving the outflow}

The bright objects and a significant fraction of the reddened objects in the WFCAM images are detected in the IRAC images, as can be seen from the IRAC $4.5-\mu \mathrm{m}$ contours around the point sources in the continuum-subtracted $\mathrm{H}_{2}$ image (Fig. 2). The most prominent among these is a bright infrared source detected near the centre of the outflow lobes in the IRAC and MIPS images. This source is saturated in the $24-\mu \mathrm{m}$ MIPS image. Its coordinates derived from the IRAC 8.0- $\mu \mathrm{m}$ image are $\alpha=17: 55: 48.91$, $\delta=-24: 40: 19.9^{2}$; it is only at a separation of $2^{\prime \prime}$ from the IRAS position. The IRAC catalog does not list the magnitudes for this source in the 3.6 and $4.5-\mu \mathrm{m}$ bands. At these wavelengths, the source appears elongated in the Spitzer images and the emission from it is blended with the emission from the outflow. For the purpose of fitting the SED, we derived the 3.6 and $4.5-\mu \mathrm{m}$ magnitudes of the outflow source from the Spitzer images. Relative photometry was performed by adopting an aperture of 8-pixel $\left(4.8^{\prime \prime}\right)$ diameter. For each band, an average zero point was derived from a few well detected and isolated point sources (which have IRAC magnitudes available) spread over the image. We derived a magnitude of $9.33 \pm 0.03$ in the $3.6-\mu \mathrm{m}$ band and $7.45 \pm 0.05$ in the $4.5-\mu \mathrm{m}$ band. The errors given are the formal errors in the zero points. Even at this aperture, the emission from the outflows will be contributing to the observed fluxes. For the SED fitting, we adopted an error of $0.18 \mathrm{mag}$.

We identify the source detected in the Spitzer data as the YSO driving the outflow "1". A deeply embedded object seen only in $K$ and $\mathrm{H}_{2}$, located near the centre of the outflow, is labelled "A" ( $\alpha=17: 55: 48.95, \delta=-24: 40: 18.31)$ in Fig. 1. "A"

${ }_{2}$ All coordinates given in this paper are in $\mathrm{J} 2000$

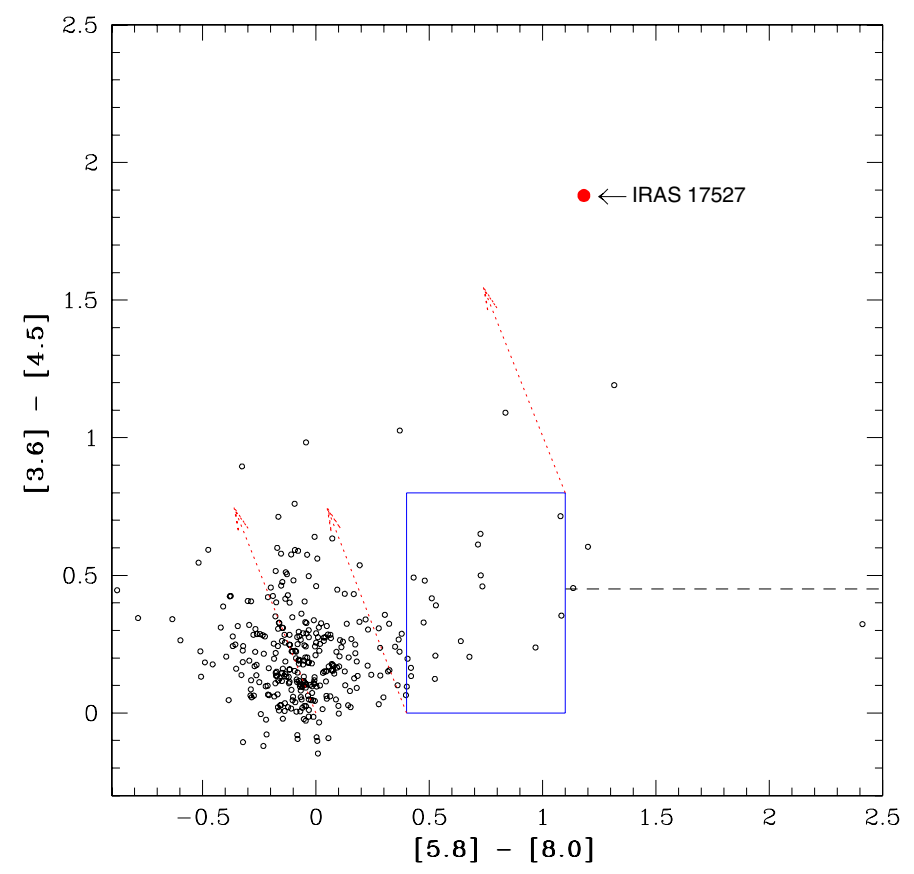

Fig. 4. The IRAC colour-colour diagram. The open (black) circles show the colours of objects detected in all four bands in a $5^{\prime} \times 5^{\prime}$ field centred on IRAS 17527. The filled (red) circle shows the outflow source. The blue rectangle shows the location of Class II sources. The dashed horizontal line shows the boundary between Class I/II objects (below) and Class I objects (above). The dotted red arrows show reddening vectors for $A_{V}=45$.

appears slightly extended in $K$ and is surrounded by nebulosity. Its coordinates are offset $1.65^{\prime \prime} \mathrm{NE}$ from that of the Spitzer source measured at $8 \mu \mathrm{m}$. Photometry within an aperture of $2^{\prime \prime}$ diameter gives a $K$ magnitue of $12.82 \pm 0.04$ for "A". It remains to be investigated through high angular resolution imaging at near- and mid-IR wavelengths to understand if " $A$ " is the nearIR counterpart of the outflow source. The $K$ magnitude of " $\mathrm{A}$ " is close to what is implied by the fit to the SED (Fig. 5). However, "A" has a positional offset from the Spitzer source, which, even though small, is significant, and the SED fitting gives an inclination of $18^{\circ}$ with respect to the line of sight for the disk axis. From the appearance of the outflow "1", the angle appears to be higher (Sect. 3.1). A higher inclination of the disk will pose a higher circumstellar extinction for the outflow source, making it too faint to detect in the near-IR. In addition, the the magnitude of "A" estimated here is likely to be contaminated by nebulosity. With these considerations, we are treating the $K$ magnitude of "A" as only an upper limit for modelling the SED of the YSO (Sect. 3.2.1).

IRAS 17527 is detected well in all mid- and far-IR bands of AKARI satellite. The coordinates given in the AKARI IRC point source catalog is only $1.2^{\prime \prime} \mathrm{NW}$ of the Spitzer position. At far-IR wavelengths, AKARI - FIS detected a bright object (AKARI-FIS-V1 1755489-244001; $\alpha=17: 55: 48.89$ $\delta=-24: 40: 0.82)$ in all 4 bands. The coordinates of the FIS detection are offset $18.75^{\prime \prime}$ towards the north of the source detected by IRC. This offset is more than the positional accuracy quoted by the AKARI point source catalogue. However, since it is the only far-IR source in the region, and the coordinates of the infrared source detected in this region by Spitzer and IRAS agree well, we ascribe the FIS detection to IRAS 17527.

The IRAC ([5.8]-[8.0], [3.6]-[4.5]) colour-colour diagram can be used to roughly identify YSOs of different evolutionary 


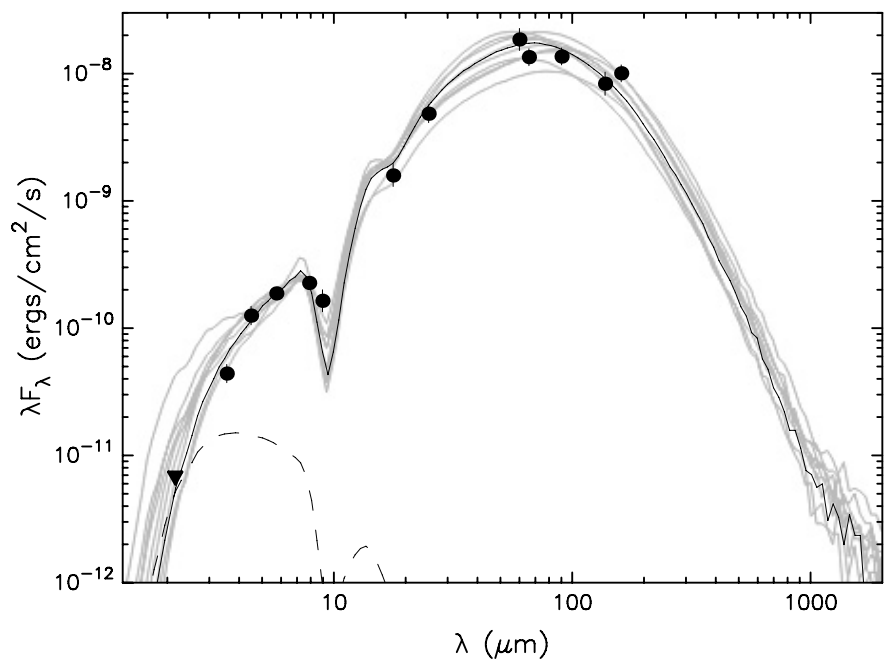

Fig. 5. The filled circles show the data from Spiter-IRAC at 3.6, 4.5, 5.8 and $8 \mu \mathrm{m}$, IRAS at 25 and $60 \mu \mathrm{m}$, and AKARI at 9, 18, 65, 90, 140 and $160 \mu \mathrm{m}$. The downward directed triangle shows the $K$-band data, which is treated as an upper limit only. The continuous line shows the best fit model and the gray lines show subsequent good fits for $\left(\chi^{2}-\chi_{\text {bestit }}^{2}\right)$ per data point $<3$. The dashed line corresponds to the stellar photosphere for the central source of the best fitting model, as it would look in the absence of circumstellar dust (but including interstellar extinction).

stages. Figure 4 shows the IRAC colours of objects, within a $5^{\prime} \times 5^{\prime}$ field around IRAS 17527. The large concentration of sources around $([5.8]-[8.0],[3.6]-[4.5])=0$ are foreground and background stars and diskless pre-main-sequence (Class III) objects. The dashed horizontal line shows the boundary between Class I/II (below) and Class I objects (above). The rectangle (0 $<([3.6]-[4.5])<0.8$ and $0.4<([5.8]-[8.0]<1.1)$ shows the location of Class II objects. The sources with $([3.6]-[4.5])>0.8$ and $([5.8]-[8.0])>1.1$ are likely to be Class I objects, which are protostars with infalling envelopes (Allen et al. 2004; Megeath et al. 2004; Qiu et al. 2008). The dotted red arrows in Fig. 4 are reddening vectors for $A_{V}=45$ calculated from the extinction law given in Mathis (1990). The location of IRAS 17527 in Fig. 4 suggests that it is a highly reddened Class I object. As seen from the SED modelling in Fig. 5, the emission from the envelope contributes significantly to the luminosity, which is expected for Class I objects.

\subsubsection{The spectral energy distribution}

The $K$ magnitude measured from the WFCAM data, magnitudes in the Spitzer bands 1-4, IRAS 25 and $60-\mu \mathrm{m}$ data and the AKARI data from 9 to $160 \mu \mathrm{m}$ were used to construct the SED of the source driving the outflow. Colour corrections were applied to the IRAS and AKARI data using the correction factors given in their respective point source catalogs (Beichman et al. 1988; Kataza et al. 2010; Yamamura et al. 2010). The SED was modelled using the on-line SED fitting tool of Robitaille et al. (2007), which uses a grid of 2D radiative transfer models presented in Robitaille et al. (2006), developed by Whitney et al. (2003, 2003b; etc.). The grid consists of 20000 YSO models with SEDs covering a wide range of stellar masses $\left(0.1-50 M_{\odot}\right)$ and evolutionary stages (from the early envelope infall stage to the late disk-only stage), each at 10 different viewing angles.

Figure 5 shows the SED of IRAS 17527 and the model fit. Table 1 shows the parameters of the best fit model. The foreground visual extinction $A_{V}$ is estimated to be $45.43 \mathrm{mag}$. The
Table 1. Results from SED fitting.

\begin{tabular}{ll}
\hline \hline Parameter & Best fit values $^{a}$ \\
\hline Stellar mass $\left(M_{\odot}\right)$ & $12.23(+1.4,-0.97)$ \\
Stellar age $(\mathrm{yr})$ & $2.08( \pm 1) \times 10^{4}$ \\
Stellar radius $\left(R_{\odot}\right)$ & $39.94(+54,-12.9)$ \\
Stellar temperature $(\mathrm{K})$ & $8093(+2000,-2600)$ \\
Disk mass $\left(M_{\odot}\right)$ & $9.79(+29,-7.3) \times 10^{-2}$ \\
Disk accretion rate $\left(M_{\odot} \mathrm{yr}^{-1}\right)$ & $4.16(+139,-3.2) \times 10^{-6}$ \\
Disk/envelope inner radius $(\mathrm{AU})$ & $5.91(+7.9,-0.83)$ \\
Disk outer radius $(\mathrm{AU})$ & $28.6(+69,-12)$ \\
Envelope mass $\left(M_{\odot}\right)$ & $1.56(+1,-0.67) \times 10^{3}$ \\
Envelope accretion rate $\left(M_{\odot} \mathrm{yr}^{-1}\right)$ & $2.42(+1.9,-0.84) \times 10^{-3}$ \\
Envelope outer radius $(\mathrm{AU})$ & $1.00(+0,-0.079) \times 10^{5}$ \\
Total Luminosity $\left(L_{\odot}\right)$ & $6.17(+2,-1.5) \times 10^{3}$ \\
Angle of inclination of the disk axis $\left(^{\circ}\right)$ & 18 \\
\hline
\end{tabular}

Notes. ${ }^{(a)}$ The values given in parenthesis are the rms of the differences, in parameter values of models with $\left(\chi^{2}-\chi_{\text {bestit }}^{2}\right)$ per data point $<3$, above and below those of the best fit model, estimated with respect to the parameter values of the best fit model. A distance of $3.23 \mathrm{kpc}$ is adopted for IRAS 17527.

fitting gives a stellar mass of $12.23 M_{\odot}$ and a total luminosity of $6.17 \times 10^{3} L_{\odot}$. The mass of the YSO and the systemic luminosity are reasonably well determined, so are the envelope parameters. However, the radius of the YSO, the disk accretion rate and the disk mass and radii have large errors. The near- and mid-IR fluxes have a significant influence on the reliable determination of these parameters. Contamination of the source magnitudes at these wavelengths due to the contribution from the outflow and from a possible companion may be the cause for the poor estimate of these parameters. Hence, photometry at high sensitivity and spatial resolution in the $2-20-\mu \mathrm{m}$ wavelength range is warranted for a more accurate determination of the stellar and disk parameters. Photometry at sub-mm wavelengths also is required for this source to better constrain the model fit.

\section{Conclusions}

1. IRAS 17527 appears to be a luminous YSO taking birth through disk accretion. A well-collimated parsec-scale outflow is discovered in IRAS 17527 in $\mathrm{H}_{2}$ line emission.

2. The outflow is seen to be bent in an "S"'-shaped fashion, suggesting a precession of the jet. There is a tentative detection of a second outflow in the $\mathrm{H}_{2}$ image. The possibility of more than one YSO in IRAS 17527 needs to be explored.

3. The $\mathrm{H}_{2}$ image exhibits a strong continuum component in the emission along the outflow, which may be caused by radiation escaping through the outflow cavity. This, in turn, reduces the radiation pressure on the accreted matter and aids growth of the central source through accretion.

4. The bulk of the emission in the direction of the outflow, observed in $K$ and the Spitzer bands, is rotated counterclockwise with respect to the direction of the outflow traced by the $\mathrm{H}_{2}$ line emission knots. It is probably a result of the precession of the jet. Spectroscopy in the the near-IR through Spitzer-IRAC bands is required for a proper understanding of this.

5. The model fit to the SED shows that the central source is probably a Class-I protostar; this is supported by its location in the Spitzer-IRAC colour-colour diagram. The YSO has a mass of $\sim 12.23 M_{\odot}$ and a total luminosity of $\sim 6.17 \times 10^{3} L_{\odot}$. 
6. The disk parameters and the disk accretion ratio are poorly determined by the SED fitting. This may be caused by the contribution to the source magnitudes from emission from the outflow and from a possible companion. Observations at high angular resolution are required in the 2-20 $\mu \mathrm{m}$ wavelength range for a more accurate determination of the source magnitudes at these wavelengths.

Acknowledgements. The UKIRT is operated by the Joint Astronomy Centre on behalf of the Science and Technology Facilities Council (STFC) of the UK. The UKIRT data presented in this paper are obtained during the UKIDSS back up time. I thank the Cambridge Astronomical Survey Unit (CASU) for processing the WFCAM data, and the WFCAM Science Archive (WSA) for making the data available. This work makes use of data obtained with AKARI, a JAXA project with the participation of ESA, and IRAS data downloaded from the SIMBAD database operated by CDS, Strasbourg, France. The Spitzer archival data are downloaded from NASA/ IPAC Infrared Science Archive, which is operated by the Jet Propulsion Laboratory, Caltec, under contract with NASA. I also thank the anonymous referee and the editor Malcolm Walmsley for their comments and suggestions which have improved the paper.

\section{References}

Allen, L. E., Calvet, N., D’Alessio, P., et al. 2004, ApJS, 154, 363

Beichman, C. A., Neugebauer, G., Habing, H. J., Clegg, P. E., \& Chester, T. J. 1988, IRAS, 1

Beuther, H., Schilke, P., Sridharan, T. K., et al. 2002, A\&A, 383, 892

Bonnell, I. A., Bate, M. R., \& Zinnecker, H. 1998, MNRAS, 298, 93

Bronfman, L., Nyman, L. A., \& May, J. 1996, A\&AS, 115, 81

Casali, M., Adamson, A., Alves de Oliveira, C., et al. 2007, A\&A, 467, 777

Davis, C. J., Gell, R., Khanzadyan, T., Smith, M. D., \& Jenness, T. 2010, A\&A, $511, \mathrm{~A} 24$
Edris, K. A., Fuller, G. A., \& Cohen, R. J. 2007, A\&A, 465, 865 Felli, M., Palagi, F., \& Tofani, G. 1992, A\&A, 255, 293

Fazio, G. G., Hora, J. L., Allen, L. E., et al. 2004, ApJS, 154, 10 Goddi, C., Moscadelli, L., Alef, W., et al. 2005, A\&A, 432, 161 Hughes, V. A., \& MacLeod, G. C. 1994, ApJ, 427, 857

Kataza, H. et al. 2010, AKARI-IRC Point Source Catalogue Release note Version 1.0

Krumholz, M. R., McKee, C. F., \& Klein, R. I. 2005, ApJ, 618, L33

Mathis, J. S. 1990, ARA\&A, 28, 37

Megeath, S. T., Allen, L. E., Gutermuth, R. A., et al. 2004, ApJS, 154, 367

Molinari, S., Brand, J., Cesaroni, R., \& Palla, F. 1996, A\&A, 308, 573

Murakami, H., Baba, H., Barthel, P., et al. 2007, PASJ, 59, S369

Neufeld, D. A., Nisini, B., Giannini, T., et al. 2009, ApJ, 706, 170

Palla, F., Brand, J., Cesaroni, R., Comoretto, G., \& Felli, M. 1991, A\&A, 246, 249

Qiu, K., Zhang, Q., Megeath, S. Th., et al. 2008, ApJ, 685, 1005

Rieke, G. H., Young, E. T., Engelbracht, C. W., et al. 2004, ApJS, 154, 25

Robitaille, T. P., Whitney, B. A., Indebetouw, R., Wood, K., \& Denzmore, P. 2006, ApJS, 167, 256

Robitaille, T. P., Whitney, B. A., Indebetouw, R., \& Wood, K. 2007, ApJS, 169, 328

Slysh, V. I., Val'tts, I. E., Kalenskii, S. V., et al. 1999, A\&AS, 134, 115

Smith, M. D. 1995, A\&A, 296, 789

Varricatt, W. P., Davis, C. J., Ramsay, S., \& Todd, S. P. 2010, MNRAS, 404, 661 van der Walt, D. J., Gaylard, M. J., \& MacLeod, G. C. 1995, A\&AS, 110, 81

Walsh, A. J., Hyland, A. R., Robinson, G., \& Burton, M. G. 1997, MNRAS, 291, 261

Whitney, B. A., Wood, K., Bjorkman, J. E., \& Cohen, M. 2003a, ApJ, 598, 1079

Whitney, B. A., Wood, K., Bjorkman, J. E., \& Wolff, M. J. 2003b, ApJ, 591, 1049

Yamamura, I., Makiuti, S., Ikeda, N., et al. 2010, AKARI-FIS Bright Source Catalogue Release note Version 1.0

Yorke, H., \& Sonnhalter, C. 2002, ApJ, 569, 846

Zhang, Q., Hunter, T. R., Brand, J., et al. 2005, ApJ, 625, 864 